\title{
Hyponatremia and cyst growth in neonatal polycystic kidney disease: a case for aquaretics?
}

\author{
Detlef Bockenhauer $^{1,2}$ (D)
}

Received: 29 December 2016 / Revised: 30 December 2016 / Accepted: 3 January 2017 /Published online: 13 February 2017

(C) IPNA 2017

\begin{abstract}
Hyponatremia is a common complication in neonatal polycystic kidney disease and is thought to be due to water retention. Aquaretics are drugs that promote free water excretion by blocking the arginine vasopressin receptor type 2 (AVPR2) in the collecting duct and thus impair urinary concentration. AVPR2 is also a key stimulant for cyclic AMP production in the collecting duct and in this way promotes cyst proliferation and pathologic kidney growth in autosomal dominant polycystic kidney disease (ADPKD). Consequently, the aquaretic tolvaptan is now used to slow down progression of ADPKD in adult patients. Whether this beneficial effect on retarding cystic disease progression also extends to recessive forms of polycystic kidney disease (PKD) is currently not known. A recent case report in Pediatric Nephrology touches on the intersecting indications for tolvaptan for both hyponatremia and cyst retardation in neonatal PKD and suggests that use for one indication may have beneficial effects on the other.
\end{abstract}

Key words Aquaretic - Tolvaptan - ADPKD · ARPKD . Hyponatremia

\section{Aquaretics and hyponatremia}

With the discovery of the arginine vasopressin receptor type 2 receptor (AVPR2) in the kidney and its dysfunction as the

\section{Detlef Bockenhauer}

d.bockenhauer@ucl.ac.uk

Centre for Nephrology, University College London, London, UK

2 Department of Pediatric Nephrology, Great Ormond Street Hospital NHS Foundation Trust, Great Ormond Street, London WC1N 3JH, UK cause of X-linked nephrogenic diabetes insipidus (NDI), AVPR2 immediately became an attractive target for drug development. Since then, the highly specific agonist 1-deamino8-D-arginine vasopressin has become available and is routinely used in the treatment of cranial NDI. More recently, antagonists have been developed, the so-called "aquaretics" or "vaptans", which are used in conditions of water excess, such as the syndrome of inappropriate antidiuretic hormone (SIADH) or congestive heart failure [1]. The development of these drugs beautifully demonstrates how the study of a rare disease (X-linked NDI, which identified the drug target) can benefit other, more common diseases.

\section{Aquaretics and polycystic kidney disease}

Autosomal dominant polycystic kidney disease (ADPKD) is the most common inherited cause of kidney failure, accounting for up to $10 \%$ of end stage kidney disease in adults [2]. Investigations into the molecular mechanisms of ADPKD identified cyclic AMP (cAMP) as a key driver for cyst and pathologic kidney growth and AVPR2 as the main stimulant for cAMP production in the collecting duct [3]. Consequently, aquaretics became an attractive drug for the treatment of ADPKD, and a large trial indeed showed that the treatment slowed cyst and kidney growth [4]. Based on these data, in some parts of the world, including the UK, tolvaptan has been approved as the first medical treatment of ADPKD in adults. A trial to investigate the effect in children is currently underway. Tolvaptan comes at a price: not only is there a risk of liver toxicity, but there is quite literally a price to pay: we recently calculated that a year's treatment of hyponatremia in the UK with one $15 \mathrm{mg}$ tablet daily cost more than $£ 30,000$ [5]. To facilitate the long-term 
treatment of ADPKD, the company has lowered the price for the ADPKD indication. This has led to the somewhat bizarre situation that tolvaptan is currently marketed by the same company under two different brand names and with two different price tags: as the more expensive SAMSCA ${ }^{\circledR}$ for the treatment of hyponatremia and as JINARC® for ADPKD.

\section{Aquaretics in the treatment of hyponatremia as a complication of PKD}

A recent case report in Pediatric Nephrology straddles the indications for the two brands and raises some interesting questions: Gilbert et al. report a case of an infant with biallelic PKD1 variants who was treated with tolvaptan $[6,7]$. In rare instances, like the reported case, the combination of a disease-causing ADPKD mutation on one allele with a hypomorphic variant on the other allele can cause a form of severe neonatal PKD that is mimicking the autosomal recessive form (ARPKD) [8]. Renal abnormalities in this baby were already noted antenatally at 17 weeks of gestation in the form of large, echobright kidneys, and she was born with massively enlarged kidneys. During the first months of life she developed typical complications of severe neonatal PKD, including hyponatremia, hypertension, edema and respiratory distress. The hyponatremia creates a management dilemma: How best to treat? Supplementation with sodium to normalize plasma sodium levels usually worsens the hypertension. Clinically, the combination of hyponatremia and hypertension suggests water excess, and thus appropriate treatment would be fluid restriction, yet this will limit caloric intake in babies. An expert consensus statement on ARPKD acknowledged this dilemma, but stated that it was unclear whether the water excess was due to excess arginine vasopressin (AVP) or tubule-interstitial dysfunction [9]. And this is where the case provides some tantalizing information: at 1-month of age, because of the hyponatremia and difficult clinical situation, the infant commenced treatment with tolvaptan-and the hyponatremia resolves. Moreover, no further growth of the kidneys over the following 11 months is noted, whereas the child otherwise grows normally and, consequently, the abdominal distension and associated respiratory distress also disappears. Unfortunately, critical pieces of information, such as osmolalities and AVP levels are not provided. While urine osmolality may not necessarily be informative, at least in adults with ADPKD [10], elevated AVP levels or its correlate co-peptin would have been helpful in supporting causality between tolvaptan treatment and not only the observed resolution of hyponatremia in this baby, but also the observed growth arrest in kidney/cyst size. The authors remark on increased AVP levels in PKD animals and suggest that levels could have been elevated in their patient due to activation of thoracic baroreceptors because of impaired cardiac filling with the severe abdominal distension, but this is obviously speculation.

\section{Aquaretics in neonatal PKD?}

Now, it is important to remember that this is a case report and thus not reliable evidence. It is possible that other groups may have tried this treatment without effect in similar cases and decided not to publish such "negative" results. However, this case report clearly makes a point for a more systematic assessment of aquaretics in severe neonatal forms of PKD. Hyponatremia is seen in up to $25 \%$ of cases of ARPKD, and hypertension is even more common. If aquaretics were to be helpful in infants with PKD and this symptom combination, this would clearly benefit their management and allow adequate feeding. At this point, it is completely unknown whether the beneficial effect of tolvaptan on cyst growth in ADPKD can be translated to ARPKD, but if treatment of hyponatremia also slowed down kidney growth in these infants, that would obviously be a welcome "side effect".

\section{Administering tolvaptan in babies}

Lastly, the authors report on a recipe for a suspension of tolvaptan. While the manufacturer is currently conducting long-term trials of tolvaptan in children, no liquid formulation suitable for long-term use has been provided, essentially excluding smaller children who are unable to take tablets from these trials. In our own off-label use, we just crush the tablet into a powder and then mix the powder in water [11], but having a more reliable suspension available would be desirable. Let us hope the manufacturer will investigate the proposed formulation for dose reliability and safety, as a liquid formulation obviously would facilitate treatment of infants.

Yet before we enthusiastically start treating hyponatremia in neonatal PKD with tolvaptan, perhaps, we should just start measuring co-peptin levels in these infants to see if there is actually any evidence of increased AVP activity which could justify the use of aquaretics. But, just for argument's sake, let us assume there was: well, which brand should we prescribe? SAMSCA® for hyponatremia? Or JINARC® for PKD? Perhaps we need a third brand with an intermediate price that covers both indications? "JINSCA" anyone?

\section{Compliance with ethical standard}

Conflict of interest The author declares no conflict of interest. 


\section{References}

1. Elhassan EA, Schrier RW (2011) Hyponatremia: diagnosis, complications, and management including V2 receptor antagonists. Curr Opin Nephrol Hypertens 20:161-168

2. Ong AC, Devuyst O, Knebelmann B, Walz G, Diseases E-EWGIK (2015) Autosomal dominant polycystic kidney disease: the changing face of clinical management. Lancet 385:1993-2002

3. Terryn S, Ho A, Beauwens R, Devuyst O (2011) Fluid transport and cystogenesis in autosomal dominant polycystic kidney disease. Biochim Biophys Acta 1812:1314-1321

4. Torres VE, Chapman AB, Devuyst O, Gansevoort RT, Grantham JJ, Higashihara E, Perrone RD, Krasa HB, Ouyang J, Czerwiec FS, Investigators TT (2012) Tolvaptan in patients with autosomal dominant polycystic kidney disease. N Engl J Med 367:2407-2418

5. Dufek S, Booth C, Carroll A, Van't Hoff W, Kleta R, Bockenhauer D (2016) Urea is successful in treating inappropriate antidiuretic hormone secretion in an infant. Acta Paediatr. doi:10.1111 /apa.13697

6. Gilbert RD, Evans H, Olalekan K, Nagra A, Haq MR, Griffiths M (2017) Tolvaptan treatment for severe, neonatal, autosomal dominant polycystic kidney disease. Pediatr Nephrol. doi:10.1007 /s00467-017-3584-9
7. Olalekan K, Fox A, Gilbert R (2016) Tolvaptan use in severe neonatal autosomal dominant polycystic kidney disease (ADPKD): The pharmaceutical challenge. Arch Dis Child 101:e2

8. Gilbert RD, Sukhtankar P, Lachlan K, Fowler DJ (2013) Bilineal inheritance of PKD1 abnormalities mimicking autosomal recessive polycystic disease. Pediatr Nephrol 28:2217-2220

9. Guay-Woodford LM, Bissler JJ, Braun MC, Bockenhauer D, Cadnapaphornchai MA, Dell KM, Kerecuk L, Liebau MC, Alonso-Peclet MH, Shneider B, Emre S, Heller T, Kamath BM, Murray KF, Moise K, Eichenwald EE, Evans J, Keller RL, Wilkins-Haug L, Bergmann C, Gunay-Aygun M, Hooper SR, Hardy KK, Hartung EA, Streisand R, Perrone R, Moxey-Mims M (2014) Consensus expert recommendations for the diagnosis and management of autosomal recessive polycystic kidney disease: report of an international conference. J Pediatr 165:611-617

10. Zittema D, Boertien WE, van Beek AP, Dullaart RP, Franssen CF, de Jong PE, Meijer E, Gansevoort RT (2012) Vasopressin, copeptin, and renal concentrating capacity in patients with autosomal dominant polycystic kidney disease without renal impairment. Clin J Am Soc Nephrol 7:906-913

11. Marx-Berger D, Milford DV, Bandhakavi M, Van't Hoff W, Kleta R, Dattani M, Bockenhauer D (2016) Tolvaptan is successful in treating inappropriate antidiuretic hormone secretion in infants. Acta Paediatr 105:e334-337 